\title{
Tendon midsubstance trauma as a means for the development of translatable chronic rotator cuff degeneration in an ovine model
}

\author{
James Johnson $^{1} \wedge$, Devin von Stade ${ }^{1 \wedge}$, Daniel $\operatorname{Regan}^{2} \wedge$, Jeremiah Easley ${ }^{3}$, Lyndah Chow ${ }^{4}$, Steven Dow $^{2,4}$, \\ Tony Romeo ${ }^{5}$, Ted Schlegel ${ }^{6}$, Kirk McGilvray $^{1 \wedge}$
}

${ }^{1}$ Orthopaedic Bioengineering Research Laboratory, Colorado State University, Fort Collins, CO, USA; ${ }^{2}$ Department of Microbiology, Immunology, \& Pathology, Flint Animal Cancer Center, Fort Collins, CO, USA; ${ }^{3}$ Preclinical Surgical Research Laboratory, Colorado State University, Fort Collins, CO, USA; ${ }^{4}$ Center for Immune and Regenerative Medicine, Department of Clinical Sciences, Colorado State University, Ft. Collins, CO, USA; ${ }^{5}$ Rothman Orthopaedic Institute, New York, NY, USA; ${ }^{\circ}$ Department of Orthopedics, University of Colorado School of Medicine, Aurora, CO, USA

Contributions: (I) Conception and design: J Johnson, D von Stade, D Regan, J Easley, T Romeo, T Schlegel, K McGilvray; (II) Administrative support: K McGilvray; (III) Provision of study materials or patients: J Johnson, D von Stade, D Regan, J Easley, K McGilvray; (IV) Collection and assembly of data: J Johnson, D von Stade, D Regan, L Chow, S Dow, K McGilvray; (V) Data analysis and interpretation: J Johnson, D von Stade, D Regan, J Easley, L Chow, S Dow, T Romeo, T Schlegel, K McGilvray; (VI) Manuscript writing: All authors; (VII) Final approval of manuscript: All authors.

Correspondence to: Kirk McGilvray, PhD. 1374 Campus Delivery, Fort Collins, CO 80523, USA. Email: kirk.mcgilvray@colostate.edu.

Background: Chronic degeneration of rotator cuff tendons is a major contributing factor to the unacceptably high prevalence of rotator cuff repair surgery failures. The etiology of chronic rotator cuff degeneration is not well understood, and current therapies are not effective, necessitating preclinical research to fill this knowledge gap. Unfortunately, current large animal models rely on enthesis disruption as a means of model generation, which is not representative of human patients with chronic rotator cuff degeneration prior to full-thickness tears. Following, the goal of this study was to develop and characterize a translational large-animal model of chronic rotator cuff degeneration without enthesis release.

Methods: A midsubstance damage model [i.e., "combed fenestration" (CF)] in adult sheep was generated by creating 16 longitudinal cuts within the top third of the infraspinatus tendon thickness. Tendon integrity was characterized through exhaustive non-destructive biomechanical stress relaxation testing [peak stress, peak load, percent relaxation, and cross-sectional area (CSA)], followed by histopathological degeneration scoring and analysis (Bonar score), histomorphological analysis of collagen organization and fatty atrophy (percent adipose area), and gene expression analyses.

Results: The CF model tendons exhibited significantly decreased mechanical properties as evidenced by decreased peak stress $(\mathrm{P}<0.025)$ and increased percent relaxation $(18$-week vs. Control, $\mathrm{P}<0.035)$ at multiple strain magnitudes and across all timepoints. At all timepoints, the CF tendons exhibited pathological changes aligned with tendon degeneration, as evidenced by increased Bonar scoring $(\mathrm{P}<0.001)$ and decreased collagen organization (6-week vs. Control, $\mathrm{P}=0.013$ ). Increases in intramuscular adipose content were also documented through histomorphology analysis $(6-$ and 18 -week vs. Control, $\mathrm{P}<0.077)$. Significant changes in gene expression were noted at all timepoints.

Conclusions: These data reveal that this new ovine CF model of chronic rotator cuff degeneration results in tendons with decreased mechanical properties, degenerative pathology characteristics, and gene expression profiles that aligned with the degenerative changes that have been noted in humans with tendinopathy. For these reasons, we believe this novel large animal model of chronic rotator cuff degeneration is a translational platform in which to test devices, therapies, and/or technologies aimed at repairing damage to the shoulder.

\footnotetext{
^ ORCID: James Johnson, 0000-0001-7465-3854; Devin von Stade, 0000-0002-2617-8283; Daniel Regan, 0000-0002-3148-1332; Lyndah Chow, 0000-0002-6413-9517; Kirk McGilvray, 0000-0003-1302-9307.
} 
Keywords: Rotator cuff; chronic degeneration; histopathology; biomechanics; gene expression

Submitted May 26, 2021. Accepted for publication Sep 02, 2021.

doi: 10.21037/atm-21-2749

View this article at: https://dx.doi.org/10.21037/atm-21-2749

\section{Introduction}

Rotator cuff tear (RCT) incidence is prominent, with some cadaver studies finding that roughly $30 \%$ of the population have tears to these tendons. Injuries to the rotator cuff decrease quality of life, with patients experiencing significant pain and decreased mobility of the arm. Unfortunately, failure rates of RCT repair surgeries are unacceptably high, with reported failure rates reaching $94 \%$ depending on exacerbating risk factors (1-4) such as chronic degeneration. Specifically, chronic degeneration of the rotator cuff tendons describes a state of diminished tissue quality which subsequently increases rate of injury and hampers the surgical repairs $(5,6)$.

Chronic degeneration, also known as tendinopathy, is a pathologic condition which describes changes to the tendon including increased cellularity, hypervascularization, surplus proteoglycan content, and decreased collagen organization (7-12). Tendinopathy originates from a combination of extrinsic and intrinsic factors, such as overuse (13), impingement (14), aging (15), changes in biology (10), and reduced mechanical properties (10). As mentioned previously, chronic degeneration is a complicating risk factor to RCT repairs. Therefore, an interventional therapy that could reverse the cascade of chronic rotator cuff tendon pathology prior to injury would revolutionize the treatment of rotator cuff injuries. Unfortunately, such a therapy has yet to be developed.

Preclinical animal testing is a vital step in the development and validation of therapies. Current animal models of chronic rotator cuff degeneration almost exclusively utilize enthesis trauma to induce pathology that replicates the hallmarks of chronic degeneration (16-23). Previous large-animal models have been implemented through a variety of surgical insults, ranging from complete transection with delayed repair (18), partial transection with delayed repair (16), and partial transection of the enthesis (24). Early models (i.e., full or partial transection with delayed repairs) produced tendon tissue with severe and reactive pathology beyond what has been noted clinically, while also generating severe tendon retraction that precludes repair. While these models play a vital role in testing devices intended at improving repair integrity, the presence of trauma at the enthesis is not representative of a chronically degenerated tendon pre-tear (i.e., older patients with a history of overuse without tendon tears). It is well known that reestablishment of the native fibrocartilaginous insertion is not currently possible (25); with tendon mechanics permanently altered following enthesis disruption. With this in mind, it is evident that these tear models do not lend themselves to the testing of therapies intended at restoring the midsubstance environment of a healthy tendon.

Therefore, the goal of this work was to generate and characterize a model of chronic tendon degeneration that does not originate from direct enthesis damage. Furthermore, the magnitude and manifestations of the histopathological and gene expression changes exhibited by this model at multiple timepoints were to be compared to a positive control (i.e., degenerated human rotator cuff tendon tissue) in order to validate and prove translatability of this model. We hypothesized that surgical micro-trauma to the mid-tendon body without direct enthesis trauma would induce pathologic changes that accurately emulate the chronic degenerative condition seen in humans, as measured by exhaustive mechanical, pathological, structural, and gene expression analyses. We present the following article in accordance with the ARRIVE reporting checklist and MDAR reporting checklist (available at https://dx.doi. org/10.21037/atm-21-2749).

\section{Methods}

The study was conducted in accordance with the Declaration of Helsinki (as revised in 2013). The study was approved by institutional ethics board of the Rush Medical University (No. 16110707-IRB01) and informed consent was taken from all the patients. Animal experiments were performed under a project license (No. 18-7854A) granted by institutional committee board of Colorado State University, in compliance with United States national or institutional guidelines for the care and use of animals. 

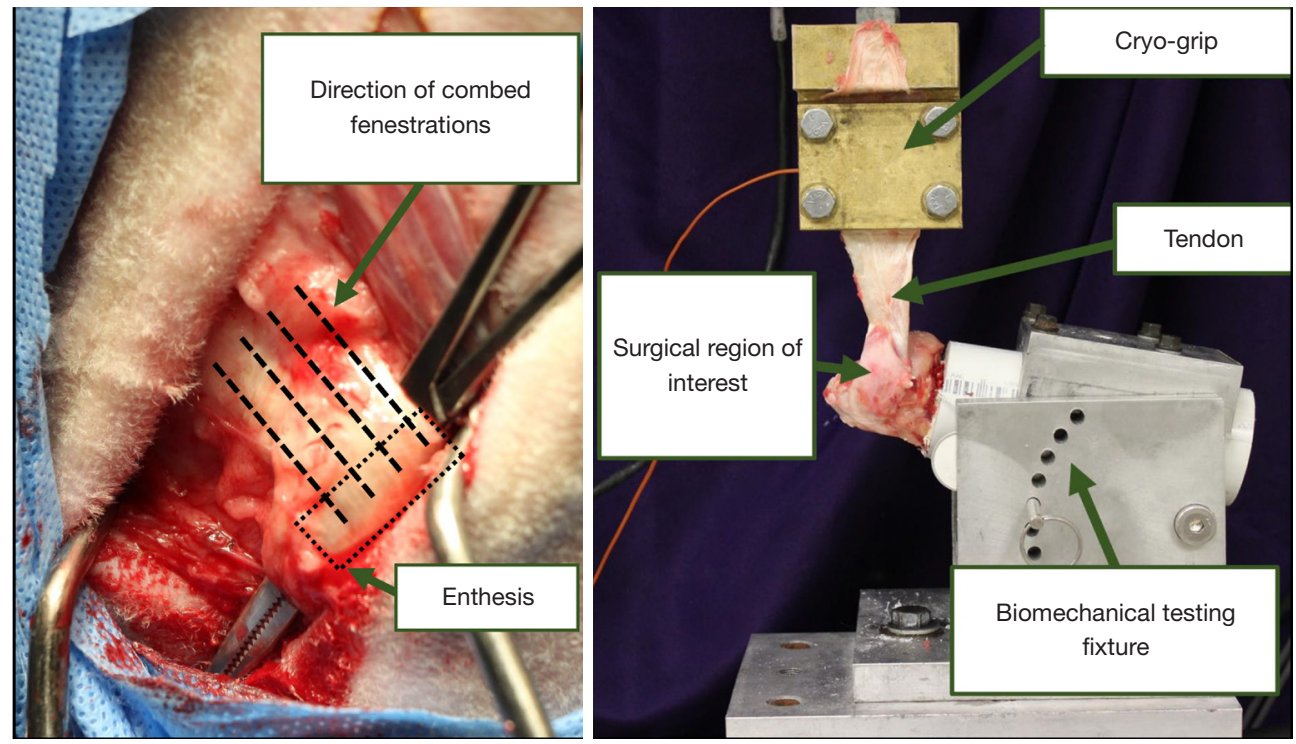

Figure 1 Photos illustrating model generation and biomechanical testing. (Left) Intra-operative image depicting the location and direction of microtrauma on the ovine infraspinatus tendon. Square box indicated the enthesis. Dotted lines represent location and direction of the combed fenestrations (only 4 of the 16 cuts are illustrated). (Right) Image of biomechanical testing (image from previously published article "Enthesis trauma as a means for the development of translatable chronic rotator cuff degeneration in an ovine model". Figure source: https://dx.doi.org/10.21037/atm-21-354; use permitted under the Creative Commons Attribution License the CC BY-NC-ND 4.0).

\section{Ovine models—surgical procedure}

Bilateral surgical insults were completed on the infraspinatus tendons in 20 Ovis aries ewes ( $\geq 3.5$ years of age) generating forty treated shoulders. Rams were excluded from this study as a result of animal husbandry concerns. The surgical models were alternated between shoulders to eliminate the potential of a left/right side bias and are detailed as follows: (I) sharp partial transection of the entheses [n=20 treated tendons-data published previously (24)] and (II) combed fenestration (CF) of the enthesis and tendon mid-substance ( $\mathrm{n}=20$ treated tendons).

The surgical procedure has been detailed previously (24). The surgical midsubstance trauma model, a.k.a., "combed fenestration", was generated by creating 16 longitudinal cuts within the top third of the infraspinatus tendon thickness (Figure 1). This insult was chosen to simulate micro-trauma of the tendon that did not result in separation of the tendon from the humerus, with the objective to replicate what is hypothesized to occur in humans with chronic degeneration prior to tendon tears. The underlying reasoning for this surgical insult was that by inducing micro-trauma on the tendon, the strain profile within the tendon would be altered, leading to similar degenerative changes that have been documented in humans clinically. Additionally, the creation of micro-trauma causing separation of the tendon collagen, extra-cellular matrix, and vasculature, would lead to a wound healing cascade, impacting tendon quality and function. Upon completion of model generation, standard surgical closure procedures were followed. The ewes were permitted to ambulate and eat ad libitum for the duration of the study. On a daily basis, sheep were monitored for all signs of complications and to evaluate incisional site healing, lameness/ambulatory function, and pain.

Sheep were sacrificed humanely at 6-, 12-, and 18-week to acquire the temporal tissue response to injury and subsequent degeneration. Immediately following sacrifice ( $<20 \mathrm{~min}$ ), the humerus-infraspinatus tendon constructs (HTCs) were harvested and subsequently isolated and denuded of soft tissues, ensuring no damage was imparted on the infraspinatus tendon. As detailed previously (24), a small portion $(5 \mathrm{~mm} \times 20 \mathrm{~mm})$ of tendon was snap frozen for the purpose of RNA sequencing.

\section{Biomechanical testing}

HTCs were tested non-destructively to determine their viscoelastic material properties as previously described (24). These properties were assessed as a means to benchmark 
the altered biomechanical properties of the tendon tissue at multiple timepoints, such that the effectiveness of future therapies could be assessed against these values. Tendon cross-sectional area (CSA) measurements were taken in triplicate proximal to the insertion (17,26-28). To ensure the highest level of stress in each tendon was reported, force was transformed to stress using the minimum of the three CSA measurements. The mean of the three CSA measurements was taken to determine the tendon overall CSA. Subsequently, the humeri were affixed in polyvinyl chloride (PVC) sleeves using a strong two-part hard casting resin (SmoothCast 321, Smooth-On, Macungie, PA, USA). PVC sleeves were gripped in a custom fixture attached to a servo-hydraulic load frame (Model 858, MTS Corp., Eden Prairie, MN, USA), enabling anatomically accurate loading of the tendon (Figure 1) (19,27). Tendons underwent a preloading phase ( $5 \mathrm{~N}$ static force, 2 minutes duration) prior to biomechanical testing to normalize viscoelastic effects (17,29-33). Following the pre-loading phase, gage length of the tendon was measured, allowing transformation of the displacement $(\mathrm{mm})$ data into stretch $(\mathrm{mm} / \mathrm{mm})$. Nondestructive stress relaxation testing was then performed on each sample, wherein a physiologically relevant stretch (i.e., 1.06 and 1.08) was applied for a duration of 100 seconds $(32,34)$. Outcome parameters included peak stress (MPa), peak force $(\mathrm{N})$, and percent relaxation (\%) (35). To allow samples to come to viscoelastic equilibrium (32), tendons were subjected to a 1,000 second recovery period between tests. Physiological saline spray was used to ensure adequate tendon hydration throughout the entire preparation and biomechanical testing processes.

\section{Histological analysis}

To determine microstructural and cellular characteristics of the tendons, samples underwent histological processing following biomechanical testing as previously described (24). Upon completion of biomechanical testing, specimens were fixed in $10 \%$ neutral buffered formalin, decalcified in $8 \%$ trifluoroacetic acid (36), and subsequently processed using standard paraffin embedding techniques. Two 5- $\mu \mathrm{m}$ thick slides were produced from each specimen, with one slide allocated to hematoxylin and eosin (H\&E) staining and the other Picro-Sirius red staining.

A blinded veterinary pathologist in residency utilized the Bonar tendinopathy scale to assess H\&E slides for: (I) tenocyte reactivity, (II) angiogenesis, (III) tendon bundle organization and polarization, and (IV) deposition of ground substance (37). All categories were graded on a 4-point scale ranging from 0 (normal/ healthy) to 3 (markedly "degenerated"/pathologic) (37). The summation of these scores was used to generate an overall degeneration score ranging from 0 (normal) to 12 (degenerated) for each specimen. These scores were assessed to enable comparison of the tendon tissue generated in this model to the positive control of chronically degenerated human tendon tissue.

\section{Histomorphological assessment of collagen fiber alignment and intramuscular adipose content}

To quantify the degree of collagen organization within the tendons, histomorphological analyses were performed as described previously (24). Briefly, polarized light microscopy in conjunction with Picro-Sirius red stained slides were assessed for collagen fiber organization, as a means to quantify the microstructural changes within the tendon collagen and to compare against the positive control of chronically degenerated human tendon tissue. The percent area of organized collagen seen within the region of interest ( $25 \mathrm{~mm}$ of tendon adjacent to insertion) was quantified using commercially available software (Image-Pro Plus, RRID:SCR_007369).

Intramuscular adipose content was also quantified through histomorphometry analysis as a means to quantify the temporal changes of fatty atrophy. Muscle biopsies from the center of the infraspinatus muscle belly were taken during dissection. Samples were fixed in $10 \%$ neutral buffered formalin and processed using standard paraffin embedding techniques. Slides were stained with hematoxylin and eosin and imaged at 40x using standard optical microscopy. The area of adipose tissue and total tissue area (excluding adipose area) were quantified (ImagePro Plus, RRID:SCR_007369). To account for differences in histological artifacts, the adipose area was normalized to the total area (i.e., the sum of the adipose area and the tissue area) providing percent adipose area. To remove area selection bias, this value was calculated at three randomly chosen uniformly sized regions across each sample. The mean of these three values was taken yielding one value representing the average percent adipose tissue area for each sample. Segmentation images were reviewed by a veterinary pathologist in residency to ensure accuracy and consistency across all samples. 
Table 1 Biomechanical and geometric properties for tendon samples

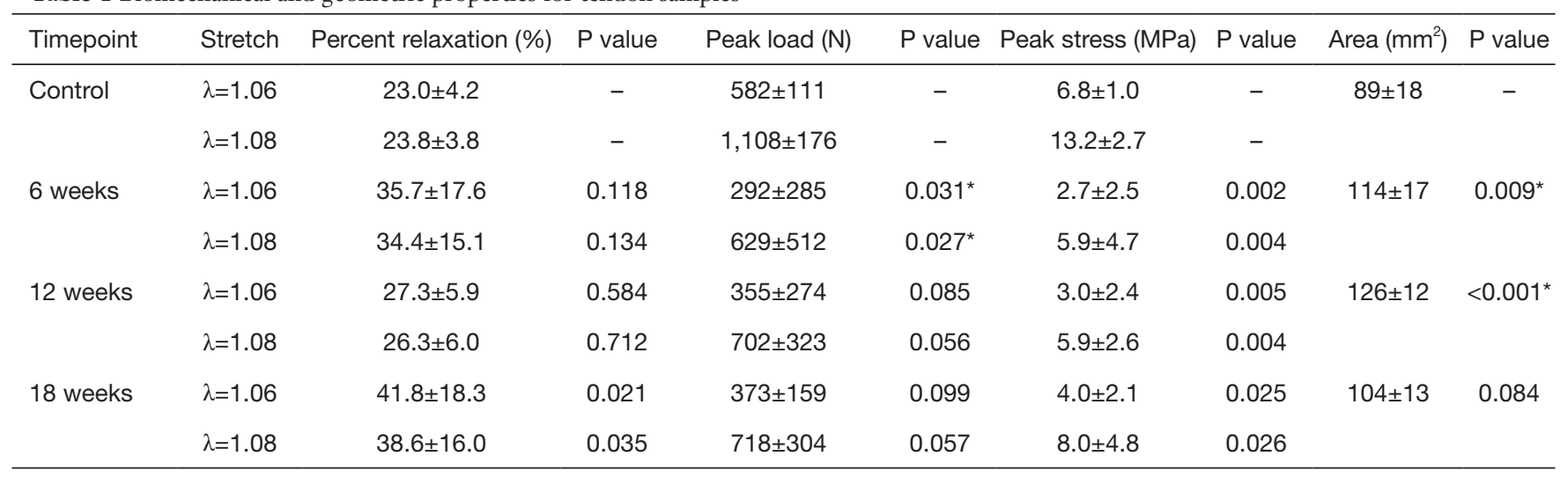

Data presented as mean \pm SD. $P$ values indicate difference with uninjured control group at same stretch level $\left({ }^{*}, P<0.05\right)$.

\section{RNA sequencing}

To determine the gene expression changes secondary to tendon injury and enable comparison to those documented in human samples previously, RNA sequencing was performed on the tendons as outlined previously (24). During fine dissection, tendon biopsies were taken and flash frozen in liquid nitrogen to prevent RNA degradation. Biopsies were pulverized then lysed (TRizol ${ }^{\mathrm{TM}}$ Invitrogen, Carlsbad, CA, USA) to precipitate RNA. The chloroform method (38) was used to isolate the RNA, with increased incubation time to account for the size of the tissue. Commercially available kits (RNeasy, Qiagen, Hilden, Germany) were then used to purify extracted RNA.

The RNA sequencing details have been described in our previous manuscript reporting the other half of this study (24). Differentially expressed genes were calculated for each timepoint compared to uninjured samples [data from previous work (24)] using Partek GSA (gene specific analysis) and non-parametric ANOVA.

\section{Statistical analysis}

Comparisons across timepoints and treatments were carried out using a one-way ANOVA followed by Fisher's post- $h o c$ test (Minitab, RRID:SCR_014483) $(\alpha=0.05)$. All data passed Anderson-Darling normality test (peak force, peak stress, percent relaxation, CSA, Bonar score, percent organized collagen, and intramuscular adipose content). A Box Cox transformation was implemented for data that failed the Levene test for equal variance (Peak Force, $\lambda=1.06$ ). Utilizing results from pilot animals, sample sizes were selected to achieve $80 \%$ statistical power. Sample numbers in each grouping were: $\mathrm{n}=6$ shoulders at the 6 - and 12 -week timepoints, and $n=7$ shoulders at the 18 -week timepoint. Uninjured ovine data from our previous publication (24) was used as a negative control for all data sets, such that changes to the tendon tissue as a result of the model could be assessed. Human samples of chronically degenerated tendons from previous work (24) were used as positive control for histopathological and histomorphological comparisons, such that this model could be adequately validated and translatability could be verified. Pearson correlation coefficients were generated as a result of correlation analyses between all outcome parameters. No specimens/samples were excluded from this study.

\section{Results}

No animals were lost during this study. No surgical complications or otherwise were noted in any animals.

\section{Biomechanical results}

A marked increase in CSA was noted in the CF tendons at all three timepoints, with percent increases as compared to the uninjured group of $28.1 \%(\mathrm{P}=0.009), 41.6 \%(\mathrm{P}<0.001)$, and $16.9 \%(\mathrm{P}=0.084)$ at the 6-, 12-, and 18-week timepoints, respectively. Moreover, the $\mathrm{CF}$ treatment groups exhibited diminished biomechanical properties (Table 1). The CF tendon samples displayed marked, significant decreases in peak stress (Figure 2), with decreases of: 60.3\% (6-week, $\lambda=1.06, \mathrm{P}=0.002$ ), 55.3\% (6-week, $\lambda=1.08, \mathrm{P}=0.004$ ), $55.9 \%$ (12-week, $\lambda=1.06, P=0.005), 55.3 \%$ (12-week, $\lambda=1.08$, $\mathrm{P}=0.004), 41.2 \%$ (18-week, $\lambda=1.06, \mathrm{P}=0.025)$, and $39.4 \%$ 

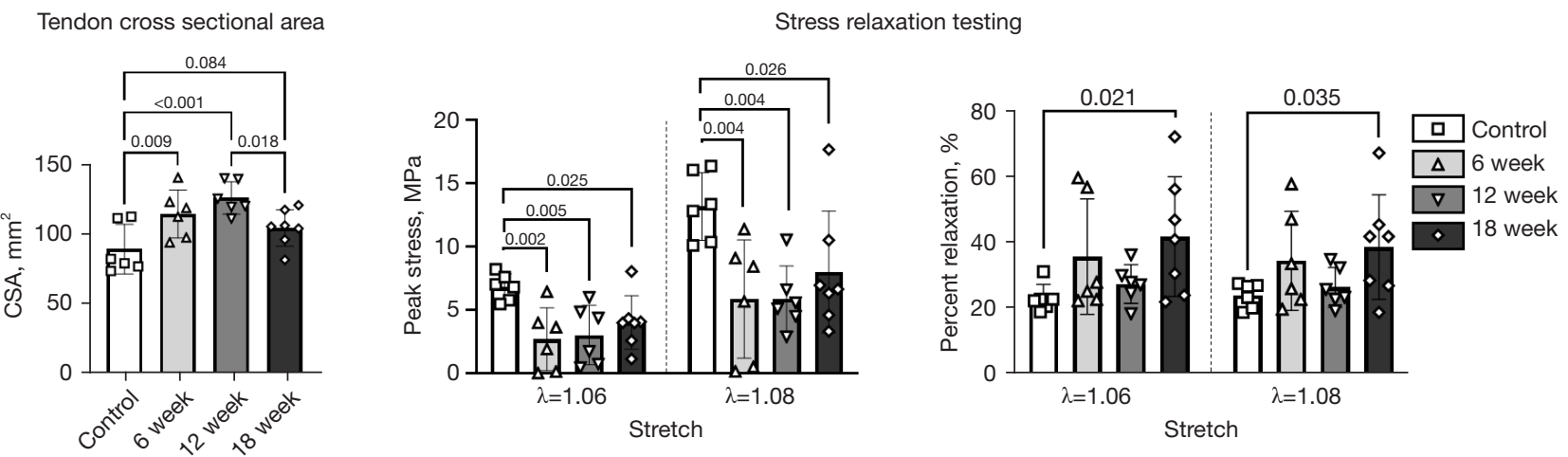

Figure 2 Biomechanical testing results. (Left) Cross-sectional area (CSA) measurements of tendon specimens. (Middle) Peak stress values from the stress-relaxation testing $(\lambda=1.06,1.08)$. (Right) Percent relaxation values from the stress-relaxation testing $(\lambda=1.06,1.08)$. $\mathrm{P}$ values are indicated for all pairwise comparisons which met the significance threshold $(\mathrm{P}<0.05)$; individual data points are marked with symbols according to their respective groups [i.e., square (Control), triangle up (6-week), triangle down (12-week), diamond (18-week)].

(18-week, $\lambda=1.08, \mathrm{P}=0.026$ ). The viscoelastic properties of the CF tendons were also altered, as evidenced by increases in percent relaxation (as compared to uninjured) at both stretch magnitudes (Figure 2), with the CF tendons exhibiting relative decreases (as compared to uninjured) of: $55.2 \%$ (6-week, $\lambda=1.06, \mathrm{P}=0.118$ ), $44.5 \%$ (6-week, $\lambda=1.08$, $\mathrm{P}=0.134), 18.7 \%$ (12-week, $\lambda=1.06, \mathrm{P}=0.584), 10.5 \%$ (12-week, $\lambda=1.08, P=0.712), 81.7 \%$ (18-week, $\lambda=1.06$, $\mathrm{P}=0.021)$, and $75.6 \%$ (18-week, $\lambda=1.08, \mathrm{P}=0.035)$.

\section{Histology results}

At all timepoints in the CF samples, the superficial third of the tendon adjacent to the enthesis was mildly thickened and markedly disrupted by disorganized collagen with loss of lamellar bundle organization and increased vascular density with noteworthy expansion of immature tenocyte populations which occasionally formed lacunae (Figure 3). A band of dense granulation tissue with prominent angiogenesis was found overlying this area and extending from the associated humeral head. Capillary clusters were prominent and often dense within the area of injury and the overlying granulation tissue (Figure 3). Enthesophyte formation of the superficial humeral head was variable.

Throughout the 6-, 12-, and 18-week timepoints there was a consistent trend toward organization of the granulation tissue overlying the injured tendon. The changes are characterized by decreased fibroblast density, smaller, less frequent blood vessels, and increased collagen disorganization with decreased tinctorial intensity. These features are consistent with a transition toward scar tissue with indistinct borders to the underlying, pre-existent tendon. Throughout the superficial tendon body in the area of injury there was a mild decrease in tendon fibroblast density over time.

The CF samples exhibited markedly increased pathologic characteristics as measured by semiquantitative Bonar scoring (Figure 4). Specifically, increases in the Bonar score were noted at all timepoints in the CF groups as compared to the uninjured group, with relative increases in Bonar scores of 173.7\% $(\mathrm{P}<0.001), 136.8 \%(\mathrm{P}<0.001)$, and $152.6 \%(\mathrm{P}<0.001)$ for the 6-, 12-, and 18-week timepoints, respectively.

\section{Histomorphology results}

Histomorphometry analysis of collagen organization revealed decreased organization in the CF samples at all timepoints (Figure 4), with relative decreases (as compared to uninjured group) of $22.9 \%(\mathrm{P}=0.189), 16.2 \%(\mathrm{P}=0.343)$, and $11.8 \%(\mathrm{P}=0.506)$ for the 6-, 12-, and 18-week groups, respectively. No significant differences were noted between the $\mathrm{CF}$ groups and the degenerated human samples. The CF samples at all timepoints demonstrated increased intramuscular adipose content as evidenced by increased percent adipose area, with relative increases in adipose area as compared to the uninjured ovine group of $56.4 \%$ $(\mathrm{P}=0.071), 30.3 \%(\mathrm{P}=0.315)$, and $53.6 \%(\mathrm{P}=0.077)$ for the 6-, 12-, and 18-week groups, respectively.

\section{$R N A$ sequencing}

The number of samples that met quality control 

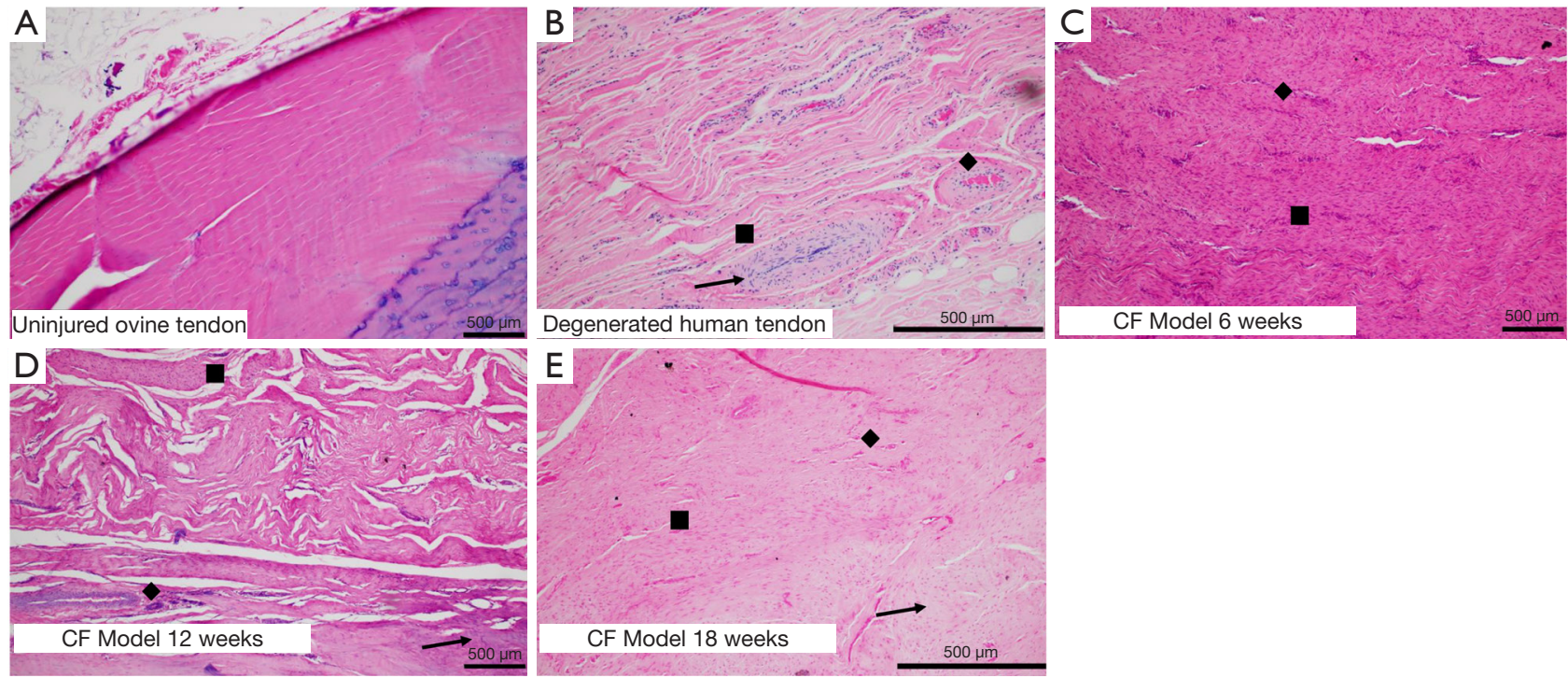

Figure 3 Representative sections of H\&E-stained specimens for all treatment groups. (A) Uninjured ovine tendon. (B) Degenerated human tendon. (C) Combed fenestration (CF) Model 6 weeks. (D) CF Model 12 weeks. (E) CF Model 18 weeks. Square indicates regions containing representative increases to tenoblast populations. Arrow indicates regions containing increased deposition of ground substance. Triangle indicates regions containing tenoblasts within lacunae. Diamond indicates representative regions with increased vascularization. For each image, the black scale bars on the bottom right corner of the image are $500 \mu \mathrm{m}$. Uninjured and human chronic degenerated group images included from previously published article "Enthesis trauma as a means for the development of translatable chronic rotator cuff degeneration in an ovine model". Figure source: https://dx.doi.org/10.21037/atm-21-354; use permitted under the Creative Commons Attribution License the CC BY-NC-ND 4.0.

Semi-quantitative pathological scoring

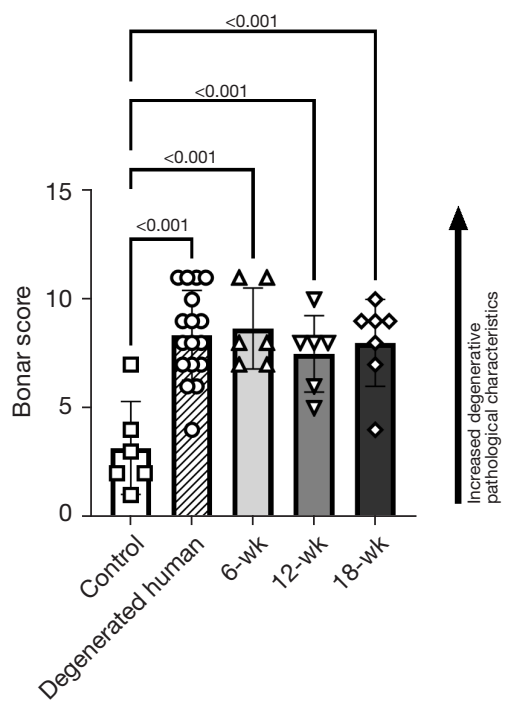

Collagen organization

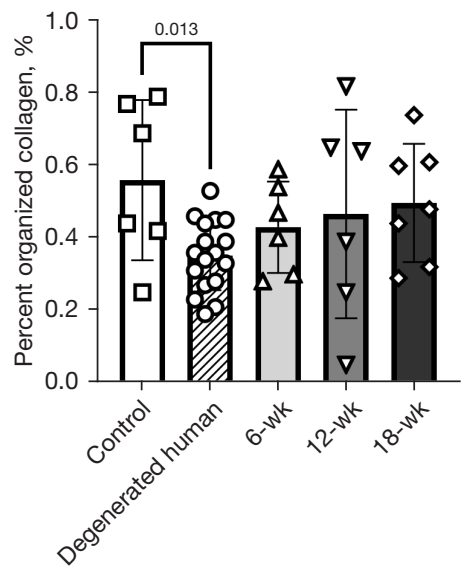

Intramuscular adipose content

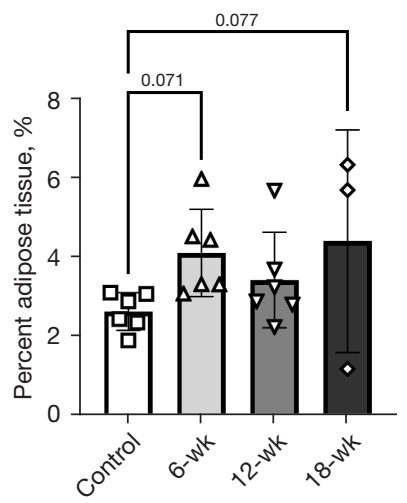

Figure 4 Pathological scoring and histomorphometry results. (Left) Overall Bonar degeneration score (Center) Percent organized collagen. (Right) Percent intramuscular adipose area. $\mathrm{P}$-values are indicated for all pairwise comparisons which met the significance threshold $(\mathrm{P}<0.05)$; individual data points are marked with symbols according to their respective groups [i.e., square (Control), circle (Degenerated Human), triangle up (6-week), triangle down (12-week), diamond (18-week)]. 

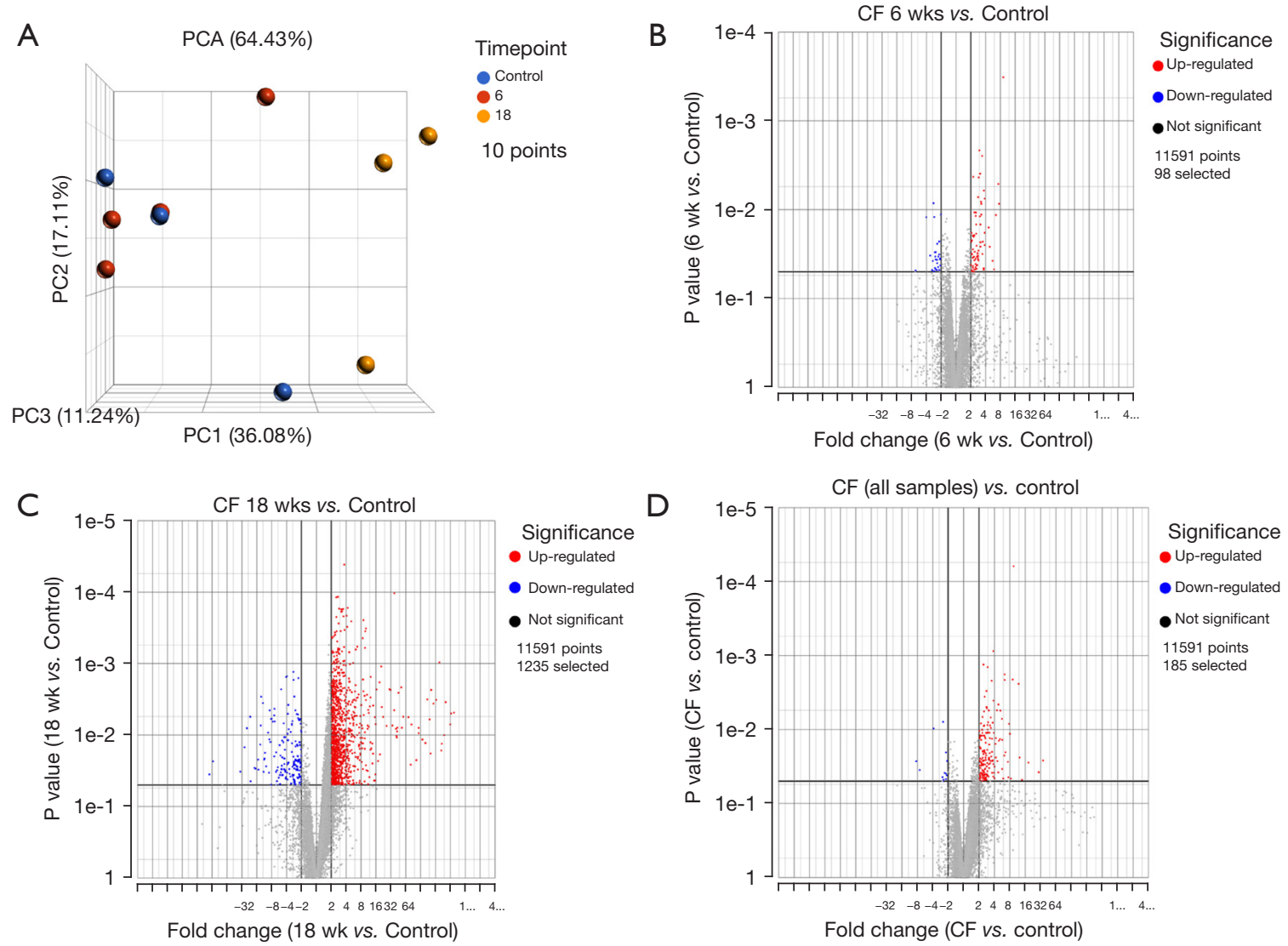

Figure 5 Gene expression changes in tendon following sharp transection. (A) Principal component analysis (PCA) plot illuminating the temporal changes in gene expression in the Combed Fenestration (CF) model and uninjured. (B) Volcano plot comparing 6-week gene expression count to uninjured. $\mathrm{X}$ axis shows fold change; $\mathrm{y}$ axis shows $\mathrm{P}$ value. Significant genes with $\mathrm{p}$ value $<0.05$ and fold change $>2$ or $<-2$ shown in bright red and green gradient with ratio scale on top right. At the six-week timepoint, there were 70 significantly upregulated genes and 28 significantly down-regulated genes. (C) Volcano plot comparing 18-week gene expression count to uninjured. 1,063 significantly up-regulated genes and 172 significantly down-regulated genes. (D) Volcano plot comparing gene expression of all CF samples count to uninjured. 172 significantly up-regulated, 13 significantly down-regulated.

specifications and underwent RNA sequencing are as follows: 6 -week $n=4,12$-week $n=0$, and 18 -week $n=3$. RNA-seq of tendon samples revealed gene expression patterns in line with tendon degeneration over the entire 18 -week observation period. As evidenced by the principal component analysis plot, the gene expression patterns of the 18-week samples appear to be the most divergent from the control group (uninjured ovine) (Figure 5A). Specifically, the significantly up-regulated + downregulated genes increased from 98 at 6 weeks post-surgery (Figure 5B) to 1,235 at 18 weeks (Figure 5C), yielding 185 across all CF samples (Figure 5D).

Gene Ontology of Biological Processes (GO BP) was evaluated to explore gene set enrichment as a means to provide a comparative baseline for future studies. Gene counts as determined via RNA-seq were tabulated and run in GSEA (version 4.1.0) using the GO_BP gene set (version 7.2). Time points were challenged individually against the uninjured group and as a pooled "injured" group (i.e., all CF samples vs. uninjured). Thirty pathways from each comparison with the highest normalized enrichment scores and false discovery rates less than $5 \%$ proceeded to graphical analysis (Figure 6). Gene sets were grouped by shared biological process ontology categories (Mouse Genome Informatics (MGI), RRID:SCR_006460). 
A CF (all samples) vs. uninjured

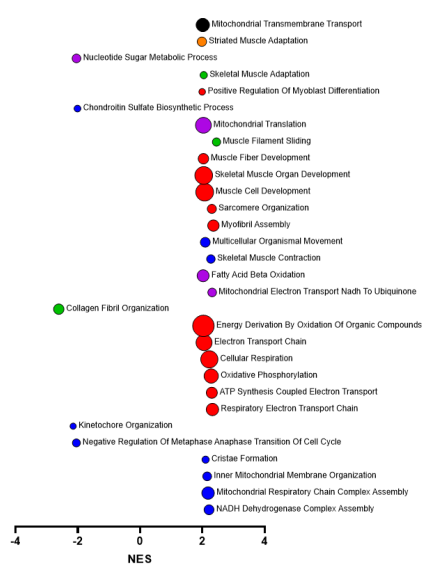

C

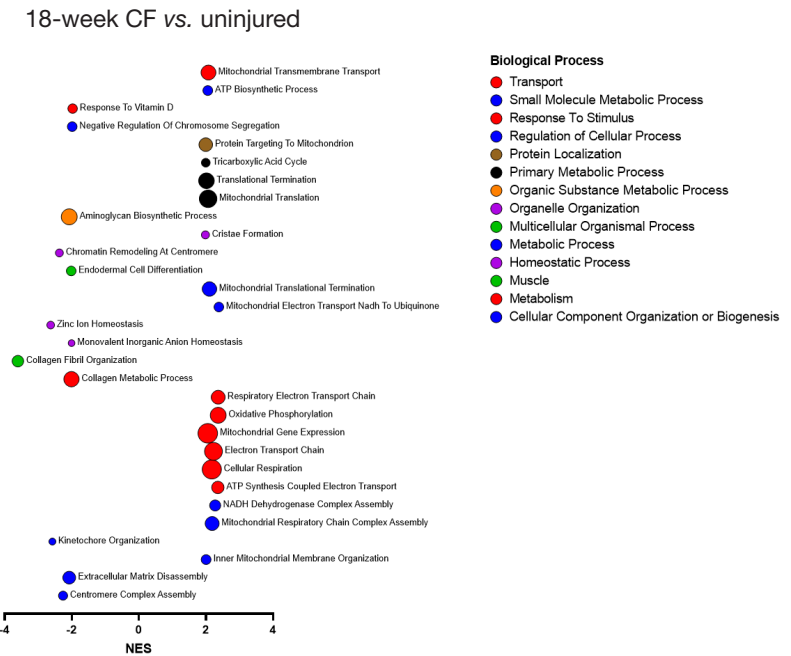

B
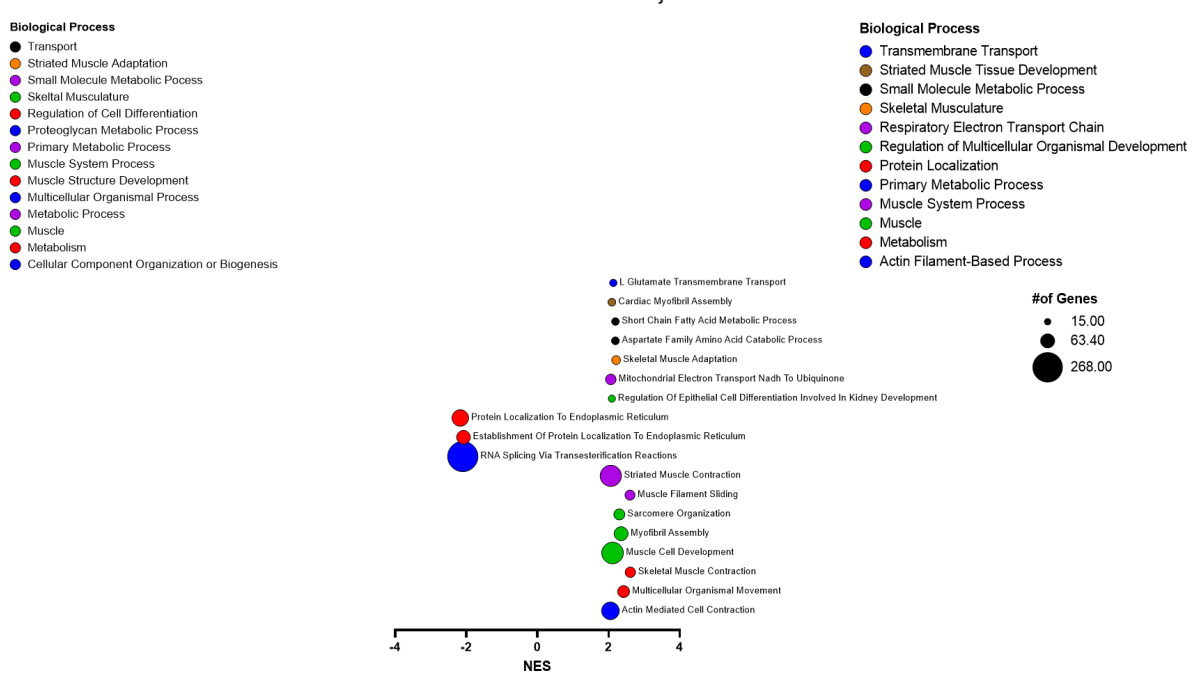

Figure 6 Bubble plot demonstrating outcomes of gene set enrichment analysis (GSEA). (A) All CF samples vs. uninjured. (B) 6-week CF vs. uninjured. (C) 18-week CF vs. uninjured. Bubble colors correspond to biological process category, bubble size according to gene count, and bubble location according to normalized enrichment scores.

\section{Correlation analysis}

An exploratory correlation analysis was performed to probe potential gene roles in other outcome parameters from the mechanical testing and pathologic scoring. To determine appropriate gene candidates, a list of genes was selected from the CF samples (both timepoints) vs. uninjured control, based on an absolute value of fold change greater than 2, leading to a potential list of 300 genes. For $n=10$ sheep, gene expression (i.e., RPKM) was compared to biomechanical and histopathological properties (i.e., peak stress, percent relaxation, CSA, Bonar score). Comparisons exceeding the significance threshold $(\mathrm{P}<0.05)$ are included in (Table 2). Specifically, there were significant correlation results (42 genes) found with collagen organization, percent relaxation, and Bonar scoring as shown in (Table 2). From this list, 19 had Pearson correlation coefficients exceeding 0.7 , indicating a strong correlation with other outcomes parameters and illuminating potential cellular pathways of 
Table 2 Correlation analyses results

\begin{tabular}{|c|c|c|c|}
\hline Outcome parameter & Gene ID & $\rho$ & $P$ value \\
\hline \multirow[t]{14}{*}{ Col. Org. (\%) } & STOX1 & 77.40 & 0.01 \\
\hline & WNT9A & 67.40 & 0.03 \\
\hline & ZNF212 & -63.80 & 0.05 \\
\hline & $C C D C 106$ & -65.90 & 0.04 \\
\hline & $R D H 13$ & -66.50 & 0.04 \\
\hline & CAPN15 & -66.90 & 0.03 \\
\hline & B3GNT8 & -69.10 & 0.03 \\
\hline & THSD4 & -0.697 & 0.03 \\
\hline & ZAP70 & -0.737 & 0.02 \\
\hline & $C D 244$ & -0.74 & 0.01 \\
\hline & CMSS1 & -0.771 & 0.01 \\
\hline & TMUB2 & -0.794 & 0.01 \\
\hline & MYO7A & -0.803 & 0.01 \\
\hline & GRAMD4 & -0.821 & 0 \\
\hline Peak stress $(\lambda=1.08)$ & THSD4 & -0.649 & 0.04 \\
\hline \multirow[t]{9}{*}{ CSA } & $B G L A P$ & 0.743 & 0.01 \\
\hline & SLC1A3 & 0.741 & 0.01 \\
\hline & FABP4 & -0.643 & 0.04 \\
\hline & TENT5B & -0.645 & 0.04 \\
\hline & $I S L R$ & -0.647 & 0.04 \\
\hline & AKAP12 & -0.659 & 0.04 \\
\hline & CBR3 & -0.688 & 0.03 \\
\hline & FN3K & -0.689 & 0.03 \\
\hline & $S 100 A 1$ & -0.737 & 0.02 \\
\hline \multirow[t]{11}{*}{ Bonar score } & PPARGC1B & 0.874 & 0 \\
\hline & B3GNT8 & 0.843 & 0 \\
\hline & $C C D C 106$ & 0.837 & 0 \\
\hline & THSD4 & 0.768 & 0.01 \\
\hline & MAP4K1 & 0.762 & 0.01 \\
\hline & NEU3 & 0.757 & 0.01 \\
\hline & TMUB2 & 0.703 & 0.02 \\
\hline & $C D 244$ & 0.687 & 0.03 \\
\hline & TFR2 & 0.67 & 0.03 \\
\hline & TRAF3IP3 & 0.669 & 0.03 \\
\hline & NUP210 & 0.667 & 0.04 \\
\hline
\end{tabular}

Table 2 (continued)
Table 2 (continued)

\begin{tabular}{cccc}
\hline Outcome parameter & Gene ID & $\rho$ & P value \\
\hline C1orf216 & 0.659 & 0.04 \\
ZDHHC12 & 0.652 & 0.04 \\
WDYHV1 & 0.646 & 0.04 \\
TRPM2 & 0.638 & 0.05 \\
PAQR8 & 0.634 & 0.05 \\
PRR7 & -0.746 & 0.01 \\
SLC6A17 & -0.752 & 0.01 \\
\hline
\end{tabular}

CSA, cross-sectional area.

tendinopathy.

The biomechanical properties of the tendons were also compared to the histopathologic scoring results. The Bonar degeneration scores were found to be strongly and significantly correlated with the peak stress at both strain magnitudes of the tendons $(\lambda=1.06: \rho=-0.65, \mathrm{P}<0.01$; $\lambda=1.08: \rho=-0.69, \mathrm{P}<0.01$; Figure 7$)$, percent relaxation $(\lambda=1.06: \rho=-0.62, \mathrm{P}<0.01 ; \lambda=1.08: \rho=-0.60, \mathrm{P}<0.01)$, and CSA of the tendons $(\rho=0.61, P<0.01)$. The peak stress was strongly and significantly correlated with the CSA of the tendons $(\lambda=1.06$ : $\rho=-0.54, \mathrm{P}=0.01 ; \lambda=1.08$ : $\rho=-0.69, \mathrm{P}<0.01$; Figure 7) and percent relaxation $(\lambda=1.06: \rho=-0.63, \mathrm{P}<0.01$; $\lambda=1.08: \rho=-0.61, P<0.01)$.

\section{Conclusions}

There are many insightful preclinical animal models of rotator cuff injury $(16,18,23,24)$; however, these models primarily rely on releasing either a portion of or the entire tendon from the humeral footprint followed by immediate or delayed reattachment. These models have fueled important discoveries in the tendon injury and repair field; unfortunately, they do not fully and/or accurately recapitulate the chronic degeneration of tendons that is observed without injury and/or trauma to the enthesis (i.e., aged population with a history of overuse resulting in chronically degenerated tendons prior to injury). Therefore, the purpose of this study was to fill this gap and generate and characterize a model of chronic rotator cuff degeneration that was not initially caused by enthesis disruption. As detailed in the results section, as early as 6-weeks persisting through the 18 -week timepoint, this model generated samples exhibiting histopathological, 

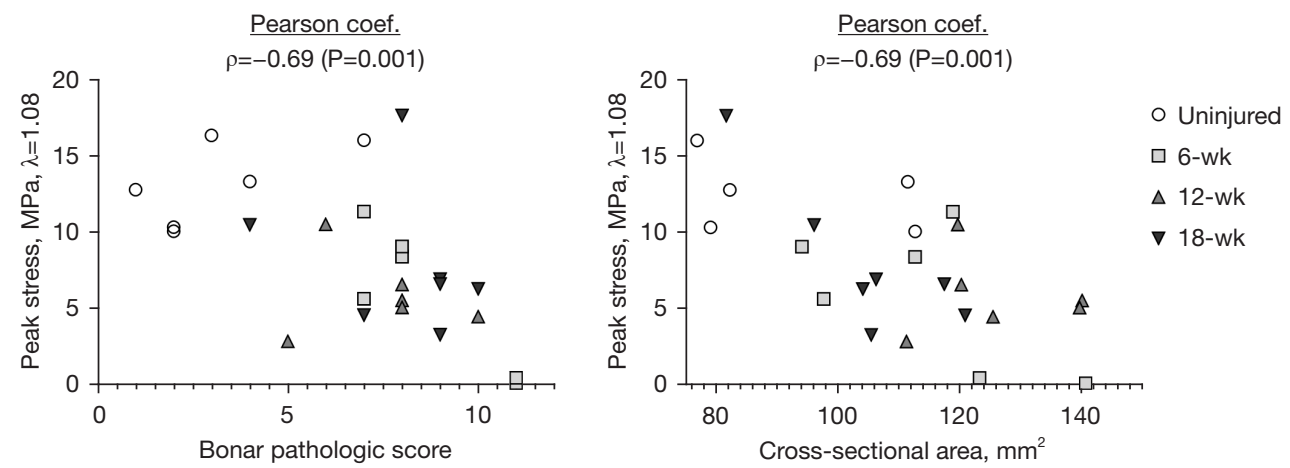

Figure 7 Representative scatterplots illustrating relationships between several outcome parameters. (Left) Peak stress was distinctly and significantly reduced in samples that demonstrated increased pathologic characteristics $(\rho=-0.69, \mathrm{P}=0.001)$. (Right) Peak stress was markedly and significantly decreased in samples with increased CSA $(\rho=-0.69, \mathrm{P}=0.001)$. Individual data points are marked with symbols according to their respective groups [i.e., circle (Control), square (6-week), triangle up (12-week), triangle down (18-week)].

collagen organization, and gene expression changes comparable to what has been documented in human tendinopathy.

Previous studies employing translational animal models to investigate RCT biomechanics have mostly performed destructive mechanical testing, reporting deleterious biomechanical changes with percent reductions in ultimate force to failure ranging from $58.1 \%$ (21) to $78.4 \%$ (39) depending on model type. Decreases in tendon biomechanical properties have also been noted in human tendon samples. Zellers et al. reported a $30.7 \%$ decrease in tendon dynamic shear modulus in Achilles tendons after injury/repair as compared to the uninjured side (40). At all timepoints, the CF model presented herein exhibited a marked decrease in peak stress ranging from $39 \%$ to $60 \%$ as compared to the uninjured group. The similarity in percent decrease of these mechanical property metrics seems to indicate that the CF model is inducing degenerative effects similar to what has been documented to occur in humans secondary to injury. Stress relaxation testing has also been performed in an ovine enthesis damage model (24), which reported deleterious changes at multiple timepoints and stretch levels, with percent decreases in peak stress ranging from $50 \%$ to $74 \%$ as compared to the uninjured group, corresponding closely with the changes in the CF model presented here. Similarly, the changes to the percent relaxation outcome parameter demonstrated by this CF model are remarkably similar to a recently characterized enthesis damage model (24), both exhibiting almost similar increases in percent relaxation secondary to injury. Considering the surgical insult was significantly different between these two models (i.e., direct enthesis damage versus direct tendon damage herein) the similarity in the decreased biomechanical properties seems to indicate that the biomechanical degenerative cascade is not damage type specific, and we hypothesis that any trauma (either acute such as a tear, or chronic such as overuse) will ultimately result in the same deleterious biomechanical changes. However, these data seem to indicate that the underlying mechanisms causing these changes (such as distributions in collagen alignment and/or gene expression) are different, possibly indicating that biomechanical testing alone is insufficient to understand the root cause of chronic tendon degeneration.

Increases in tendon CSA have also been reported previously. Derwin et al. reported marked increases in tendon CSA in a canine acute transection and repair model, ranging from $84 \%$ to $303 \%$ (20). Likewise, similar increases have been reported in an ovine model without full tendon release, with increases ranging from $58 \%$ to $63 \%$ (24). Additionally, increases in human tendon CSA following injury have been documented clinically. Zellers et al. reported a $525 \%$ increase in Achilles tendon CSA at 12 -week post-repair from full-thickness tear (40). Interestingly, this CF model demonstrated only modest increase in CSA comparatively (17\% to $42 \%$ depending on timepoint), these results demonstrate that full-thickness tears, as compared to tendon overuse, result in more pronounced geometric changes. This insight should have clinical utility in possibly determining the root cause of degeneration as the imaging of tendons and the resultant "thickening" observed, is a common parameter used to 
determine the level of degeneration in human patients $(41,42)$.

The CF ovine tendons demonstrated pathologic changes consistent with human tendinopathy (Figure $3 B-3 E$ ). Pathologic attributes noted across the CF tendon samples and timepoints are similar to those that have been described previously in human samples $(12,43-45)$. Characteristic pathologic traits exhibited across all timepoints include disorganized collagen throughout the tendon body, increased vascular density, and marked expansion of tenoblast populations. The decrease in collagen organization that was qualitatively described by the pathologist was quantified through histomorphometry, illuminating a mean decrease in collagen organization of $16.9 \%$ across the CF groups at the three timepoints normalized to the uninjured group. Similar changes in collagen organization have been noted previously, with an ovine enthesis damage model reporting a mean decrease in collagen organization of $27.0 \%$ across multiple timepoints as well (24). Furthermore, all of the pathologic changes exhibited by the CF samples were similar in magnitude and manifestation to what has been described previously in an ovine enthesis damage model (24). Again, considering the differences of initial surgical insults between the two models, the similarity in manifestation of histopathological changes indicates that the degenerative cascade is not damage type specific. This observation is further supported by the existence of pathological changes to the entirety of the tendon substance, even in regions that did not experience surgical insult.

This model and others $(16,20,21,23)$ have demonstrated clearly that injury to one isolated portion of the tendon can cause changes to the entire bone-tendon-muscle construct. Additionally, this work has quantitatively demonstrated that the changes secondary to tendon injury are not isolated to the tendon. Histomorphometry analysis of the intramuscular adipose content within the infraspinatus muscle belly illuminated large relative increases in adipose content as early as 6 weeks (56.4\% increase) and persisting through the 18 -week timepoint (67.5\% increase). Previous research has shown similar changes in muscle quality following injury, with one study having shown increases in pro-adipogeneic gene expression and adipocyte quantity in an acute ovine injury model (46). Similar reductions in associated muscle quality are a hallmark of human chronic degeneration, with assessment of fatty atrophy via magnetic resonance imaging being a primary method of diagnosis of rotator cuff injury, degeneration, and decreased surgical outcomes $(47,48)$.

The samples in this study were semi-quantitatively graded following the Bonar pathologic grading scheme, which assigns numeric values to pathologic features associated with chronic tendon degeneration (37). The mean Bonar scores for the CF tendon groups at all timepoints were within 1 point of the degenerated human control group $( \pm 10.2 \%)$. There was a strong and statistically significant correlation between the cellular activity/pathologic characteristics (encompassed in the Bonar scoring scheme) and the biomechanical performance of the tendons. Specifically, the Bonar scores of the CF tendons had strong correlations with the peak stress, percent relaxation, and CSA of the tendons (Figure 7). Knowing collagen is the primary load-carrying constituent of tendons and also a key component graded in the Bonar scheme, these results are not surprising. However, these data highlight the importance of the microstructural composition and organization to the performance of the tendon.

Gene expression patterns across the 6- and 18-week timepoints in this model embodied many of the changes documented in literature regarding injured human rotator cuff tendons. Previous work by Jelinsky et al. [dataset] (49) measured global gene expression patterns in diseased human tendons obtained intraoperatively. Their data contains findings from many tendons, including the supraspinatus and subscapularis. Upon isolating the results from the lesioned supraspinatus tendons and normalizing to the unlesioned subscapularis tendons using the GEO2R tool [Gene Expression Omnibus (GEO), RRID:SCR_005012], it was apparent the CF tendons exhibited similar overall gene expression patterns. Specifically, the CF samples had a $51.7 \%$ and $53.2 \%$ match in gene expression pattern across the entire transcriptome for the 6- and 18-week timepoints, respectively. Furthermore, the 6-week CF samples matched the collagen expression of the lesioned human tendon samples across 11 out of the 17 collagen genes, while the 18-week CF samples only matched expression with 4 of the same collagen genes. These results highlight the temporal changes in gene expression patterns secondary to tendon injury and provide reasoning to believe this model is an appropriate platform with which to investigate chronic rotator cuff degeneration. Additionally, these data provide the necessary information to illuminate potential target pathway(s) for therapy designs.

Ongoing work includes investigation of human cadaver tissue with the same analysis methodologies implemented 
herein, with the goal of enabling direct comparison between the two species. It is our hope that by investigating the pathologic and gene expression changes in a representative cohort of human samples with inferior mechanical performance, the underlying cellular pathways responsible for chronic degeneration may be illuminated, providing valuable insight for scientists working on therapeutics to arrest chronic degeneration.

While previous large animal translational models of chronic rotator cuff degeneration have been successfully implemented, this model separates itself as it does not rely on enthesis disruption yet is still capable of achieving similar degenerative pathology as what has been documented in humans. For this reason, it is our belief that this model will provide a valuable research platform to test biological therapies aimed at arresting/reversing the degeneration cascade prior to injury. Additionally, due to similarity in tendon size to the human rotator cuff tendons, this model is particularly well suited as a platform to assess scaffolds intended to improve repair outcomes for full tears of chronically degenerated tendons. Through comprehensive testing, we have demonstrated similar pathology, similar decreases in collagen organization, increased intramuscular adipose content, and similar gene expression patterns as what has been documented in humans. For these reasons, we believe that CF of the ovine infraspinatus tendon results in a translatable model of chronic rotator cuff degeneration.

\section{Acknowledgments}

The authors would like to thank Brad Nelson and Eileen Hackett for assistance with the surgeries. The authors would also like to thank Cecily Broomfield and Lauren Berens for assistance with the histology.

Funding: This work was supported by internal funding provided by the Orthopaedic Bioengineering Research Laboratory and the Preclinical Surgical Research Laboratory at Colorado State University, and was supported by the National Institute of Health [K01OD022982 and L30 TR002126 to DPR].

\section{Footnote}

Reporting Checklist: The authors have completed the ARRIVE reporting checklist and the MDAR reporting checklist. Available at https://dx.doi.org/10.21037/atm-212749
Data Sharing Statement: Available at https://dx.doi. org/10.21037/atm-21-2749

Conflicts of Interest: All authors have completed the ICMJE uniform disclosure form (available at https://dx.doi. org/10.21037/atm-21-2749). Dr. TR receives royalties from devices/products from Arthrex, Inc. and Atreon Orthopaedics. He also has been paid to present in the last 12 months by Arthrex, Inc. He is a paid consultant for Arthrex, Inc. and Atreon Orthopaedics. He has stock/stock options in Paragen. Dr. TS reports grants from Arthrex Education Grant, grants from Gemini Mountain Education Grant, outside the submitted work; and Stock/stock options in the following companies: Kaleo, PRIVIT, Avenu and NASH. The other authors have no conflicts of interest to declare.

Ethical Statement: The authors are accountable for all aspects of the work in ensuring that questions related to the accuracy or integrity of any part of the work are appropriately investigated and resolved. The study was conducted in accordance with the Declaration of Helsinki (as revised in 2013). The study was approved by institutional ethics board of the Rush Medical University (No. 16110707-IRB01) and informed consent was taken from all the patients. Animal experiments were performed under a project license (No. 18-7854A) granted by institutional committee board of Colorado State University, in compliance with United States national or institutional guidelines for the care and use of animals.

Open Access Statement: This is an Open Access article distributed in accordance with the Creative Commons Attribution-NonCommercial-NoDerivs 4.0 International License (CC BY-NC-ND 4.0), which permits the noncommercial replication and distribution of the article with the strict proviso that no changes or edits are made and the original work is properly cited (including links to both the formal publication through the relevant DOI and the license). See: https://creativecommons.org/licenses/by-nc-nd/4.0/.

\section{References}

1. Galatz LM, Ball CM, Teefey SA, et al. The outcome and repair integrity of completely arthroscopically repaired large and massive rotator cuff tears. J Bone Joint Surg Am 2004;86:219-24.

2. Cole BJ, McCarty LP 3rd, Kang RW, et al. Arthroscopic 
rotator cuff repair: prospective functional outcome and repair integrity at minimum 2-year follow-up. J Shoulder Elbow Surg 2007;16:579-85.

3. Harryman DT 2nd, Mack LA, Wang KY, et al. Repairs of the rotator cuff. Correlation of functional results with integrity of the cuff. J Bone Joint Surg Am 1991;73:982-9.

4. Meyer M, Klouche S, Rousselin B, et al. Does arthroscopic rotator cuff repair actually heal? Anatomic evaluation with magnetic resonance arthrography at minimum 2 years follow-up. J Shoulder Elbow Surg 2012;21:531-6.

5. McIntyre LF, Bishai SK, Brown PB 3rd, et al. PatientReported Outcomes After Use of a Bioabsorbable Collagen Implant to Treat Partial and Full-Thickness Rotator Cuff Tears. Arthroscopy 2019;35:2262-71.

6. Fukuda $\mathrm{H}$. The management of partial-thickness tears of the rotator cuff. J Bone Joint Surg Br 2003;85:3-11.

7. Longo UG, Ronga M, Maffulli N. Achilles tendinopathy. Sports Med Arthrosc Rev 2009;17:112-26.

8. Samiric T, Parkinson J, Ilic MZ, et al. Changes in the composition of the extracellular matrix in patellar tendinopathy. Matrix Biol 2009;28:230-6.

9. Ibrahim M, Kartus JT, Steigen SE, et al. More tendon degeneration in patients with shoulder osteoarthritis. Knee Surg Sports Traumatol Arthrosc 2019;27:267-75.

10. Seitz AL, McClure PW, Finucane S, et al. Mechanisms of rotator cuff tendinopathy: intrinsic, extrinsic, or both? Clin Biomech (Bristol, Avon) 2011;26:1-12.

11. Dean BJ, Franklin SL, Carr AJ. A systematic review of the histological and molecular changes in rotator cuff disease. Bone Joint Res 2012;1:158-66.

12. Longo UG, Franceschi F, Ruzzini L, et al. Histopathology of the supraspinatus tendon in rotator cuff tears. Am J Sports Med 2008;36:533-8.

13. VanBaak K, Aerni G. Shoulder Conditions: Rotator Cuff Injuries and Bursitis. FP Essent 2020;491:11-6.

14. Neer CS 2nd. Anterior acromioplasty for the chronic impingement syndrome in the shoulder: a preliminary report. J Bone Joint Surg Am 1972;54:41-50.

15. Rudzki JR, Adler RS, Warren RF, et al. Contrast-enhanced ultrasound characterization of the vascularity of the rotator cuff tendon: age- and activity-related changes in the intact asymptomatic rotator cuff. J Shoulder Elbow Surg 2008;17:96S-100S.

16. Easley J, Johnson J, Regan D, et al. Partial Infraspinatus Tendon Transection as a Means for the Development of a Translational Ovine Chronic Rotator Cuff Disease Model. Vet Comp Orthop Traumatol 2020;33:212-9.

17. McGilvray KC, Lyons AS, Turner AS, et al. Shoulder
Tendon Repair Biomechanics Using a Polyurethane Patch in a Chronic Ovine Defect Model. Proceedings of the ASME 2007 Summer Bioengineering Conference. ASME 2007 Summer Bioengineering Conference. Keystone, Colorado, USA. June 20-24, 2007. pp. 853-4.

18. Coleman SH, Fealy S, Ehteshami JR, et al. Chronic rotator cuff injury and repair model in sheep. J Bone Joint Surg Am 2003;85:2391-402.

19. Santoni BG, McGilvray KC, Lyons AS, et al. Biomechanical analysis of an ovine rotator cuff repair via porous patch augmentation in a chronic rupture model. Am J Sports Med 2010;38:679-86.

20. Derwin KA, Baker AR, Codsi MJ, et al. Assessment of the canine model of rotator cuff injury and repair. J Shoulder Elbow Surg 2007;16:S140-8.

21. Derwin KA, Codsi MJ, Milks RA, et al. Rotator cuff repair augmentation in a canine model with use of a woven polyL-lactide device. J Bone Joint Surg Am 2009;91:1159-71.

22. Melrose J, Smith MM, Smith SM, et al. Altered stress induced by partial transection of the infraspinatus tendon leads to perlecan (HSPG2) accumulation in an ovine model of tendinopathy. Tissue Cell 2013;45:77-82.

23. Smith MM, Sakurai G, Smith SM, et al. Modulation of aggrecan and ADAMTS expression in ovine tendinopathy induced by altered strain. Arthritis Rheum 2008;58:1055-66.

24. Johnson J, von Stade D, Regan D, et al. Enthesis trauma as a means for the development of translatable chronic rotator cuff degeneration in an ovine model. Ann Transl Med 2021;9:741.

25. Derwin KA, Galatz LM, Ratcliffe A, et al. Enthesis Repair: Challenges and Opportunities for Effective Tendon-toBone Healing. J Bone Joint Surg Am 2018;100:e109.

26. Noyes FR, Butler DL, Grood ES, et al. Biomechanical analysis of human ligament grafts used in knee-ligament repairs and reconstructions. J Bone Joint Surg Am 1984;66:344-52.

27. Easley J, Puttlitz C, Hackett E, et al. A prospective study comparing tendon-to-bone interface healing using an interposition bioresorbable scaffold with a vented anchor for primary rotator cuff repair in sheep. J Shoulder Elbow Surg 2020;29:157-66.

28. McGilvray KC, Santoni BG, Turner AS, et al. Effects of (60)Co gamma radiation dose on initial structural biomechanical properties of ovine bone--patellar tendon-bone allografts. Cell Tissue Bank 2011;12:89-98.

29. France EP, Paulos LE, Harner CD, Straight CB. Biomechanical evaluation of rotator cuff fixation methods. 
Am J Sports Med 1989;17:176-81.

30. Schlegel TF, Hawkins RJ, Lewis CW, et al. The effects of augmentation with Swine small intestine submucosa on tendon healing under tension: histologic and mechanical evaluations in sheep. Am J Sports Med 2006;34:275-80.

31. Rodeo SA, Arnoczky SP, Torzilli PA, et al. Tendon-healing in a bone tunnel. A biomechanical and histological study in the dog. J Bone Joint Surg Am 1993;75:1795-803.

32. Sverdlik A, Lanir Y. Time-dependent mechanical behavior of sheep digital tendons, including the effects of preconditioning. J Biomech Eng 2002;124:78-84.

33. Troyer KL, Puttlitz CM. Nonlinear viscoelasticty plays an essential role in the functional behavior of spinal ligaments. J Biomech 2012;45:684-91.

34. Paxton JZ, Hagerty P, Andrick JJ, et al. Optimizing an intermittent stretch paradigm using ERK1/2 phosphorylation results in increased collagen synthesis in engineered ligaments. Tissue Eng Part A 2012;18:277-84.

35. Liao J, Vesely I. Relationship between collagen fibrils, glycosaminoglycans, and stress relaxation in mitral valve chordae tendineae. Ann Biomed Eng 2004;32:977-83.

36. Broomfield C, Meis N, Johnson J, et al. Optimization of ovine bone decalcification for increased cellular detail: a parametric study. J Histotechnol 2021. [Epub ahead of print]. doi: 10.1080/01478885.2021.1951053.

37. Cook JL, Feller JA, Bonar SF, et al. Abnormal tenocyte morphology is more prevalent than collagen disruption in asymptomatic athletes' patellar tendons. J Orthop Res 2004;22:334-8.

38. Sikes KJ, Li J, Gao SG, et al. TGF-b1 or hypoxia enhance glucose metabolism and lactate production via HIF1A signaling in tendon cells. Connect Tissue Res 2018;59:458-71.

39. Hee CK, Dines JS, Dines DM, et al. Augmentation of a rotator cuff suture repair using rhPDGF-BB and a type I bovine collagen matrix in an ovine model. Am J Sports Med 2011;39:1630-9.

40. Zellers JA, Pohlig RT, Cortes DH, et al. Achilles tendon

Cite this article as: Johnson J, von Stade D, Regan D, Easley J, Chow L, Dow S, Romeo T, Schlegel T, McGilvray K. Tendon midsubstance trauma as a means for the development of translatable chronic rotator cuff degeneration in an ovine model. Ann Transl Med 2021;9(21):1616. doi: 10.21037/atm21-2749 cross-sectional area at 12 weeks post-rupture relates to 1-year heel-rise height. Knee Surg Sports Traumatol Arthrosc 2020;28:245-52.

41. Tuckman GA. Abnormalities of the long head of the biceps tendon of the shoulder: MR imaging findings. AJR Am J Roentgenol 1994;163:1183-8.

42. Buck FM, Grehn H, Hilbe M, et al. Degeneration of the long biceps tendon: comparison of MRI with gross anatomy and histology. AJR Am J Roentgenol 2009;193:1367-75.

43. Sethi PM, Sheth CD, Pauzenberger L, et al. Macroscopic Rotator Cuff Tendinopathy and Histopathology Do Not Predict Repair Outcomes of Rotator Cuff Tears. Am J Sports Med 2018;46:779-85.

44. Nakajima T, Rokuuma N, Hamada K, et al. Histologic and biomechanical characteristics of the supraspinatus tendon: Reference to rotator cuff tearing. J Shoulder Elbow Surg 1994;3:79-87.

45. Roßbach BP, Gülecyüz MF, Kempfert L, et al. Rotator Cuff Repair With Autologous Tenocytes and Biodegradable Collagen Scaffold: A Histological and Biomechanical Study in Sheep. Am J Sports Med 2020;48:450-9.

46. Luan T, Liu X, Easley JT, et al. Muscle atrophy and fatty infiltration after an acute rotator cuff repair in a sheep model. Muscles Ligaments Tendons J 2015;5:106-12.

47. Mellado JM, Calmet J, Olona M, et al. Surgically repaired massive rotator cuff tears: MRI of tendon integrity, muscle fatty degeneration, and muscle atrophy correlated with intraoperative and clinical findings. AJR Am J Roentgenol 2005;184:1456-63.

48. Gladstone JN, Bishop JY, Lo IK, et al. Fatty infiltration and atrophy of the rotator cuff do not improve after rotator cuff repair and correlate with poor functional outcome. Am J Sports Med 2007;35:719-28.

49. Jelinsky SA, Rodeo SA, Li J, et al. Regulation of gene expression in human tendinopathy. BMC Musculoskelet Disord 2011;12:86. 